\title{
New drug lead from Madagascar's rainforests
}

DOI:

10.1038/nrd2256
Given the global spread of

multidrug-resistant malaria, new chemotherapeutic agents are urgently needed. Most antimalarial drug discovery is aimed at the asexual blood stages. However, Carraz et al., reporting in PLoS Medicine, have now identified a potent lead compound that is specifically active against the obligatory primary liver stage of malaria. Targeting this stage has the advantage that it is limited both in duration and parasite numbers, thereby minimizing the parasite's chances to develop drug resistance.

The search for new antimalarials led the authors to the rainforests of Madagascar, where 229 plant species with reported antimalarial properties were systematically analysed.

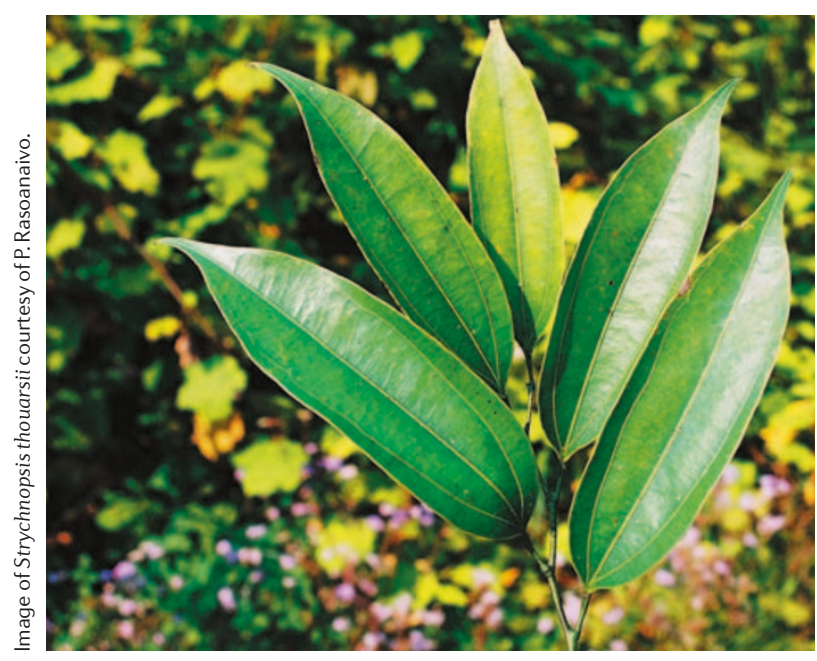

One of the plants, Strychnopsis thouarsii, was selected for further investigation as its bark is the sole ingredient of a widely used remedy reputed to provide protection specifically against malaria. As the active compound in the traditional decoction did not seem to be active against the blood stage of malaria, the authors established primary mouse and human liver cell (hepatocyte) cultures that allowed the analysis of the hepatic invasion stage of the malaria parasite, a stage that precedes the pathogenic erythrocytic stage.

Successive chromatographic separation led to the isolation of a pure, active, novel morphinan compound, which was named tazopsine, that has specific inhibitory action against liver but not blood stages of Plasmodium yoelii. Its antimalarial activity was shown to be due to growth retardation of the early developmental stages of the hepatic parasite.

Simple chemical modification of tazopsine led to the compound $\mathrm{N}$-cyclopentyl (NCP)-tazopsine, which demonstrated an improved toxicity profile, and conferred full protection from malarial challenge in a mouse malaria model. $\mathrm{IC}_{50}$ values and therapeutic indices did not differ substantially from primaquine, the only licensed drug with specific activity against the hepatic stage of malaria. However, primaquine and its derivatives are hampered significantly by toxicity and a risk of haemolysis in individuals with a glucose-6-phosphate dehydrogenase deficiency, a condition common in malaria endemic regions. Therefore NCP-tazopsine presents as an excellent lead candidate for compounds with improved pharmacological characteristics.

The discovery of this new class of molecules could lead to the development of a true causal prophylactic drug. The fact that, to date, all antimalarials with prophylactic activity also inhibit the erythrocytic stage precludes mass administration of these drugs, as the cyclical multiplication of malarial parasites in the blood results in extremely high parasite loads, which puts high pressure on the selection and dissemination of drugresistant parasites. However, a drug solely active against the liver stage would exert only mild selective pressure, as a single infective bite leads to only a few dozen parasites that usually persist for less than 2 weeks. Tazopsine might prove to be an invaluable lead compound in the effort to reduce the global burden of malaria.

\section{Alexandra Flemming}

ORIGINAL RESEARCH PAPER Carraz, M. et al. A plant-derived morphinan as a novel lead compound active against malaria liver stages. PLoS Med. 3, e513 (2006) 